hematemesis/occult gastrointestinal hemorrhages which has increased the incidence of haemoptysis in our study at $30,95 \%$.

Conclusion: Although IPH is a pulmonary disease, the respiratory syndrome is present on a second level, anemia being the primary symptom in our cases.

1312

\section{PULMONARY INFECTIONS IN CHILDREN WITH CYSTIC FIBROSIS}

\section{D.T. Qosaj}

\section{Pulmonology Department, University Clinical Centre of Kosova, Prishtina, Albania}

Introduction: Cystic Fibrosis (CF) is the most common inherited life shortening disease among white population but also appears among other ethnic groups and races. CF is autosome recesive disease and in Europe the most frequent mutation is delta F508.Disease is polysistemic and invols in first place respiratory system,gastrointestinal system,failure to thrive and other signs.

Aim of study: Presentation of cases treated in our ward,some clinical features focusing in most frequent infections which we found in our patients. during period of 2000-2008.

Material and methods: For the tudy we used patients records, outpatients records, hematologic laboratory findings, microbiology analyses, chest $X$ ray.

Results: We treat 32 children with diagnose as CF and $19(59.3 \%)$ are female and $13(40.6 \%)$ are male. Under 1 year of age are 8 children(25\%), from 1 till 5 years of age are18 children $(56.2 \%)$, from 5 till 10 years are 4 children(12.5\%) and above 10 years 2 children(0.62\%). Our diagnose was based in clinical features,sweat test and genetic mutation. In 6 children we had a positive family history(18\%). Frequency of their admission is as follows: 10 children(31.2\%) were admitted once a year, 9 children(28.1\%) we admitted 2 or 3 times a year and 16 children $(50 \%)$ were admitted more than three times during one year. Infectons that we isolated in ourchildren is follows:at 3 children(9.3\%)was isolated Streptococcus pneumonae, at 5 children(15.6\%) Echeria coli,at 3 children (9.3\%):Klebsiella, at 4 children(12.5\%) :Staphylococcus aureus,at 2 children(6.2\%) :Pseudomonas aeruginosa, at 2 children(6.2\%):Citrobacter,1 child(3.1\%) had
Branhamella.At other children the culture was sterile.

Conclusion: Presented cases realize that the infection is the common reason for exacerbation and admission in hospital. The agents are common as we found in literature.

Keywords: Cystic fibrosis,infection

1313

\section{ENDOTRACHEAL TUBE SIZE AND PLACEMENT IN NEONATES}

\author{
J.E. Tan'1, A. Foran², S. Ryan ${ }^{3}$ \\ ${ }^{1}$ Paediatrics-Neonates, ${ }^{2}$ Neonatology, ${ }^{3}$ Radiology, \\ Rotunda Maternity Hospital, Dublin, Ireland
}

Introduction: In neonates, accurate endotracheal tube (ET) size and placement is essential for adequate ventilation and surfactant delivery. Malposition of ET may cause asymmetrical surfactant distribution,pneumothorax, tracheal oesophageal perforation and aspiration.

Objectives: To assess: 1)ET placement 2) knowledge of correct ET placement and size in neonates.

\section{Methods:}

1. Chest X-rays (CXRs) of intubated infants were reviewed and verified by consultant radiologist JanMar 2010.

2. Questionnaires were distributed to consultants, NCHDs and NICU nurses in Rotunda and nationally during a week of duty in National Neonatal Transport Service. When 2 answers were selected, both smaller ET size and shorter ET length was chosen.

\section{Results:}

1. In total 203 CXRs were reviewed. $70 \%$ showed ETs were placed in the upper and middle $3^{\text {rd }}$ of the trachea. Only 4\% ETs were placed in the right main bronchus. No major complications noted.

2. In total 51 questionnaires were completed, 31 from tertiary unit and 20 from peripheral. From tertiary hospital, all consultants answered correctly, 10/13 NCHDs and 8/14 NICU nurses answered correctly. From the peripheral hospitals, all consultants answered correctly, 6/15 NCHDs and $1 / 3$ NIU nurses answered correctly. The variance 